\title{
Clinical and Epidemiological Characteristics of Hospitalized COVID-19 Patients in Hormozgan, Iran: A Retrospective, Multicenter Study
}

\author{
Mehdi Hasani Azad, MD'; Farid Khorrami, $\mathrm{PhD}^{2}$; Mitra Kazemi Jahromi, $\mathrm{MD}^{3}$; Nader Alishan Karami, $\mathrm{PhD}^{2}$; Mehraban Shahi, \\ $\mathrm{PhD}^{2}$; Nasrin Davari Dolatabadi, PhD²; Golnaz Sadat Mousavi, MSc'; Abbas Sheikhtaheri, PhD $^{4,5^{*}}$ \\ ${ }^{1}$ Infectious and Tropical Diseases Research Center, Hormozgan Health Institute, Hormozgan University of Medical Sciences, Bandar \\ Abbas, Iran \\ ${ }^{2}$ Health Information Technology, Faculty of Paramedicine, Hormozgan University of Medical Sciences, Bandar Abbas, Iran \\ ${ }^{3}$ Endocrinology and Metabolism Research Center, Hormozgan University of Medical Sciences, Bandar Abbas, Iran \\ ${ }^{4}$ Health Management and Economics Research Center, Health Management Research Institute, Iran University of Medical Sciences, \\ Tehran, Iran \\ ${ }^{5}$ Department of Health Information Management, School of Health Management and Information Sciences, Iran University of \\ Medical Sciences, Tehran, Iran
}

\begin{abstract}
Background: To better manage the COVID-19 pandemic, it is necessary to carefully study information about patients with COVID-19.

Objective: To report clinical and epidemiological characteristics of COVID-19 patients in southern Iran.

Methods: This cross-sectional retrospective study was conducted based on data extracted from the COVID-19 registry of Hormozgan. Data from patients with confirmed COVID-19 based on CT-scan results or real-time reverse transcriptase-polymerase chain reaction (RT-PCR) results until September 25, 2020, were analyzed for this study (2351 inpatients). We reported demographics, signs and symptoms on admission, comorbidities, and treatments, as well as clinical outcomes, hospital stay, and intensive care unit (ICU) admission.

Results: Most of patients were men $(1235 / 2351 ; 52.5 \%)$ and the most common signs and symptoms included cough (1343/2351; $57.1 \%)$, shortness of breath $(1224 / 2351 ; 52.1 \%)$ and fever. The most common comorbidities included hypertension $(410 / 2351$ $(17.4 \%)$, diabetes $(343 / 2351 ; 14.6 \%)$ and chronic cardiac disease $(282 / 2351 ; 12 \%)$. Also, 228 patients $(9.7 \%)$ were hospitalized in the ICU. The mortality rate was $12.5 \%$ (295/2351) among all patients and $64.5 \%$ (147/228) in ICU wards, respectively. The number of cases with comorbidities including hypertension, chronic cardiac disease, diabetes, chronic neurological disorders, chronic kidney disease, chronic hematologic disease, malignant neoplasm, moderate or severe liver disease, dementia and fauvism in the ICU was significantly higher than the general wards.

Conclusion: Most characteristics of our patients were similar to those reported in other studies; however, our patients were younger and suffered from a less severe disease. The mortality rate in the ICU was higher than other studies.

Keywords: COVID-19, Epidemiology, Hormozgan, Iran

Cite this article as: Hasani Azad M, Khorrami F, Kazemi Jahromi M, Alishan Karami N, Shahi M, Davari Dolatabadi N, et al. Clinical and epidemiological characteristics of hospitalized COVID-19 patients in Hormozgan, Iran: a retrospective, multicenter study. Arch Iran Med. 2021;24(5):434-444. doi: 10.34172/aim.2021.62
\end{abstract}

Received: January 23, 2021, Accepted: February 27, 2021, ePublished: May 1, 202

\section{Introduction}

A series of pneumonia cases with unknown etiology appeared in Wuhan, China, in December 2019. The clinical symptoms were very similar to those of viral pneumonia. ${ }^{1}$ However, more precise research studying the lower respiratory tract led to the discovery of a new virus, later named coronavirus 2019. ${ }^{2}$ Being highly contagious for the human-to-human infection and the rapid spread of the virus attracted global attention. Soon enough, the World Health Organization (WHO) declared the coronavirus 2019 disease (COVID-19) as an emergency for public health and international concern. ${ }^{3,4}$

Iran is one of the countries with the highest cases of
COVID-19. As of October 6, 2020, there have been 479000 confirmed cases and 27000 deaths. ${ }^{5}$ To manage COVID-19 data, Hormozgan University of Medical Sciences (HUMS), Iran, established a regional registry program (RCovidRH) in March $2020^{6}$ and started to collect the related data. Although the number of studies addressing COVID-19 is increasing in the world, these studies are mainly from China, the US, and European countries, ${ }^{7-16}$ few studies from developing countries such as Iran have also reported the clinical and epidemiological features of the disease. In the present report of RCovidRH, ${ }^{6}$ we characterized the features of patients with COVID-19 admitted to hospitals affiliated to HUMS in the Hormozgan province. 


\section{Materials and Methods}

\section{Study Design and Population}

This retrospective study was carried out based on the data obtained from the RCovidRH. ${ }^{6}$ This registry has been collecting data related to patients with a definite or suspected diagnosis of COVID-19 for both outpatients and hospitalized patients referring to 20 hospitals and healthcare facilities throughout Hormozgan province in southern Iran since March 25, 2020. There are 14 cities in Hormozgan in which more than 1,750,627 people live. RCovidRH has already collected the data from more than 5000 patients. We extracted the data related to hospitalized patients with a definite diagnosis of COVID-19 according to CT-scan or real-time reverse transcriptase-polymerase chain reaction (RT-PCR) results until September 25, 2020.

\section{Data Collection}

RCovidRH collects data both prospectively and retrospectively. The variables collected in the RCovidRH were selected based on the International Severe Acute Respiratory and Emerging Infection Consortium (ISARIC), the US Center for Disease Prevention and Control (CDC), and WHO case reporting forms. ${ }^{17-19}$ The registry extracts the required data from patients' medical records as well as PCR lab test results from the reference laboratory of the province assigned for the diagnosis of COVID-19. Two trained nurses supervised by an infectious diseases specialist review the medical records, and subsequently fill out the data extraction forms, and finally register them in a web-based software. Quality control staff in the registry control the quality of the data continuously and provide required feedback for the collection of shortcomings and completion of data. More details were provided in an earlier study. ${ }^{6}$ For this study, we extracted data of 2393 hospitalized patients with a definite diagnosis of COVID-19 from the registry. There were 42 re-admissions. For this study, the researchers solely considered the admission leading to the worst outcome (death); otherwise, the first admission was considered for the analysis. Finally, we analyzed the data from 2351 inpatients.

\section{Outcomes}

The main outcome was intensive care unit (ICU) admission. We also reported demographics, signs and symptoms on admission, comorbidities, treatments, clinical outcomes (recovery/death), and length of hospital stay.

\section{Data Analysis}

We used frequency and percentage for non-numerical data, and mean, median, and interquartile range (IQR) for numerical variables. Age categories were made based on previous studies. ${ }^{9,20}$ We used the Pearson Chi-Square or Fisher's Exact Test for non-numerical variables, and the t-test or Mann-Whitney for numerical variables for the normally distributed data. Normality of distribution was tested with the Kolmogorov-Smirnov test. The analyses were carried out using SPSS software (edition 25) and $P<0.05$ was considered as the level of significance.

\section{Results}

\section{Demographic and Social Characteristics}

Table 1 shows the demographic and social characteristics of the 2351 patients. The mean age was $47.02 \pm 20.4$ years (Min-Max: below 1 year and 99 years). Most of the patients were men $(52.5 \%)$. A total of 228 patients $(9.7 \%)$ were hospitalized in the ICU. There was a history of traveling 14 days before initiation of symptoms in $2 \%$ of patients. The percentage of healthcare staff infected by the disease was $2.7 \%$.

\section{Clinical Characteristics}

The most common comorbidities included hypertension (17.4\%), chronic cardiac disease (12\%), diabetes with or without complications (14.6\%), and asthma (5.1\%). Moreover, most patients did not have any history of smoking, using alcohol, or drug addiction. ICU patients suffered from significantly more comorbidities including hypertension ( $28.5 \%$ vs. $16.3 \%)$, chronic cardiac disease (21.9\% vs. $10.9 \%)$, diabetes with complications (14\% vs. $8 \%$ ), diabetes without complications ( $14.5 \%$ vs. $5.1 \%)$, chronic neurological disorders (9.6\% vs. $3.8 \%)$, chronic kidney disease ( $7.5 \%$ vs. $3 \%)$, chronic hematologic disease (3.9\% vs. $1.9 \%)$, malignant neoplasm ( $4.8 \%$ vs. $1.8 \%)$, moderate or severe liver disease (3.9\% vs. $1.1 \%)$, dementia $(1.3 \%$ vs. $0.2 \%)$ and fauvism $(2.6 \%$ vs. $0.7 \%)$. Other comorbidities were not significantly different between ICU and non-ICU patients.

The most common symptoms on admission included cough $(57.1 \%)$, fever (45.1\%), shortness of breath $(52.1 \%)$, fatigue (18.1\%), muscle aches (17.7\%) and chills (11.3\%). Although there was no significant difference for most symptoms between ICU and non-ICU patients, cough, seizure, shortness of breath, fever, fatigue, headache, lost sense (of smell or taste), and vertigo were significantly different between ICU and non-ICU patients (Table 2, Figures 1 and 2).

\section{Vital Signs and Laboratory Results}

According to the available vital signs and laboratory results, the values of temperature, blood pressure, platelets, APTT, sodium and potassium did not show any significant difference between ICU and non-ICU patients. The levels of hematocrit, hemoglobin, lymphocyte, and oxygen saturation of ICU patients were significantly lower than non-ICU patients while the reverse was true for other laboratory and vital sign values (Table 3).

\section{Treatments}

In total, $47.1 \%$ of the patients underwent oxygen therapy. Moreover, this procedure was applied for $95.6 \%$ of ICU patients $(P<0.0001)$. Non-invasive and invasive ventilation were respectively used for $4.5 \%$ and $6.8 \%$ of 
Table 1. Demographics and Social Characteristics of Hospitalized Patients Infected With COVID-19

\begin{tabular}{|c|c|c|c|c|}
\hline \multirow[t]{2}{*}{ Variables } & $\begin{array}{c}\text { ICU Patients } \\
(\mathrm{n}=228 ; 9.7 \%)\end{array}$ & $\begin{array}{l}\text { Non-ICU Patients } \\
(\mathrm{n}=2123 ; 90.3 \%)\end{array}$ & $\begin{array}{l}\text { Total Patients } \\
(\mathrm{n}=2351)\end{array}$ & \multirow[t]{2}{*}{$P$ Value } \\
\hline & No. $(\%)$ & No. $(\%)$ & No. $(\%)$ & \\
\hline \multicolumn{5}{|l|}{ Age } \\
\hline$<10$ & $20(8.8)$ & $79(3.7)$ & $99(4.2)$ & \multirow{8}{*}{$<0.001^{*}$} \\
\hline 10-19 & $5(2.2)$ & $80(3.8)$ & $85(3.6)$ & \\
\hline 20-29 & $11(4.8)$ & $250(11.8)$ & 261 (11.1) & \\
\hline 30-39 & $28(12.3)$ & $448(21.1)$ & $476(20.2)$ & \\
\hline $40-49$ & $26(11.4)$ & 359 (16.9) & 385 (16.4) & \\
\hline $50-59$ & $30(13.2)$ & $302(14.2)$ & $332(14.1)$ & \\
\hline $60-69$ & 43 (18.9) & 339 (16) & $382(16.2)$ & \\
\hline$\geq 70$ & $65(28.5)$ & 265 (12.5) & $330(14)$ & \\
\hline Mean $( \pm S D),(y)$ & $53.12 \pm 24.4$ & $46.37 \pm 19.8$ & $47.02 \pm 20.4$ & $<0.001 *$ \\
\hline Median (Q1, Q3) & $58.5(38,72)$ & $45(32,61)$ & $46(33,62)$ & $<0.001 *$ \\
\hline Min, Max & $<1,99$ & $<1,94$ & $<1,99$ & - \\
\hline Sex, Male & $136(59.6)$ & 1099 (51.8) & 1235 (52.5) & $0.024^{*}$ \\
\hline Pregnant & $8(3.5)$ & $65(3.1)$ & $73(3.1)$ & 0.069 \\
\hline Travel (14ds before symptom), Yes & $3(1.3)$ & $44(2.1)$ & $47(2.0)$ & $0.001 *$ \\
\hline Animal contact, Yes & $2(0.9)$ & $18(0.8)$ & $20(0.9)$ & $0.004^{*}$ \\
\hline Health care worker, Yes & $7(3.1)$ & $56(2.6)$ & $63(2.7)$ & 0.701 \\
\hline
\end{tabular}

*Significant difference

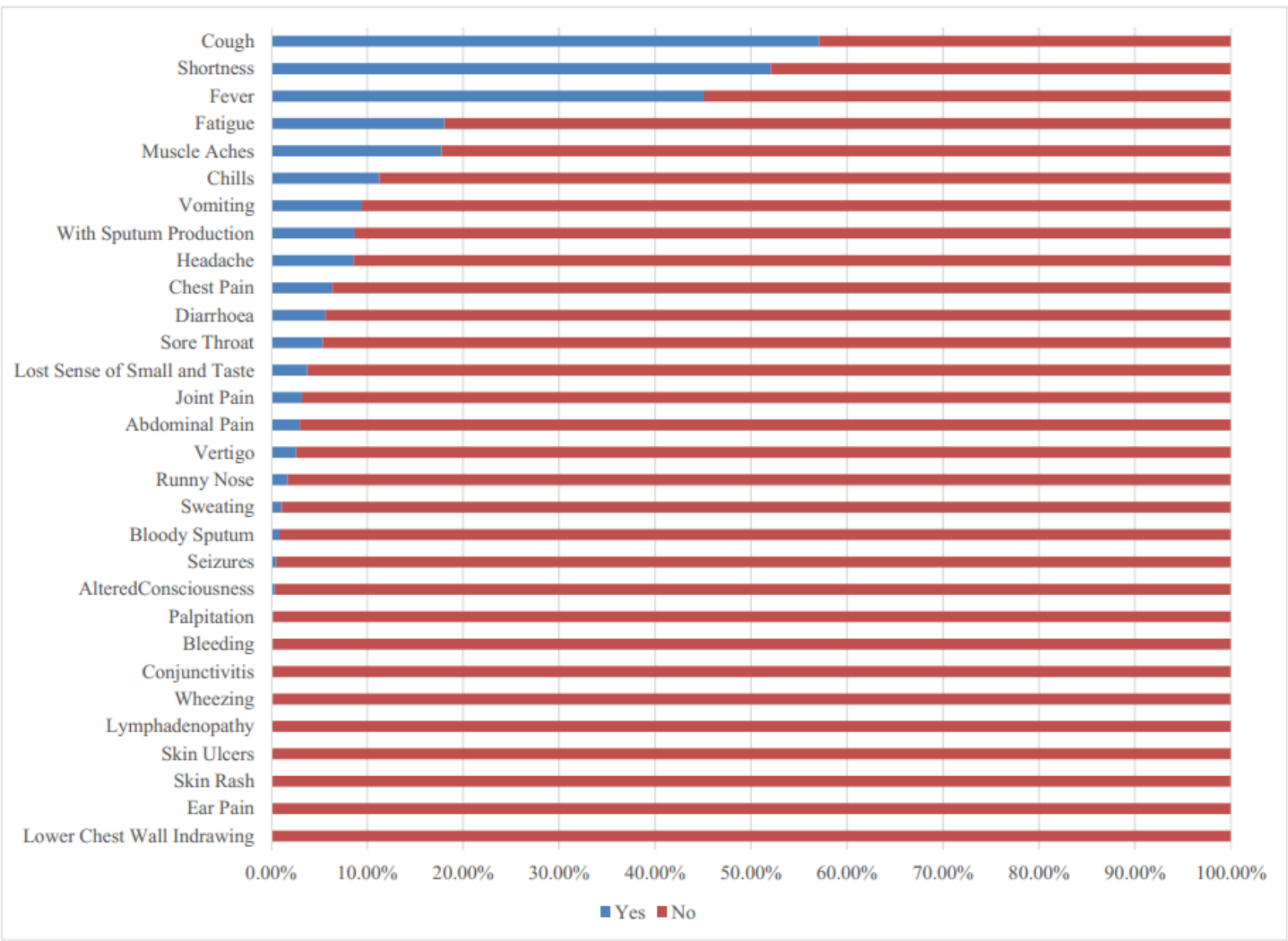

Figure 1. Distribution of Symptoms among Patients. 
Table 2. Clinical Characteristics of Hospitalized Patients Infected With COVID-19

\begin{tabular}{|c|c|c|c|c|}
\hline \multirow[t]{2}{*}{ Variables } & $\begin{array}{l}\text { ICU Patients } \\
(\mathbf{n}=\mathbf{2 2 8})\end{array}$ & $\begin{array}{l}\text { Non-ICU Patients } \\
(n=2123)\end{array}$ & $\begin{array}{c}\text { Total Patients } \\
(\mathrm{n}=2351)\end{array}$ & \multirow[t]{2}{*}{$P$ Value } \\
\hline & No. $(\%)$ & No. $(\%)$ & No. $(\%)$ & \\
\hline \multicolumn{5}{|l|}{ Comorbidities } \\
\hline Hypertension & $65(28.5)$ & $345(16.3)$ & $410(17.4)$ & $<0.0001^{*}$ \\
\hline Chronic cardiac disease & $50(21.9)$ & $232(10.9)$ & $282(12.0)$ & $<0.0001^{*}$ \\
\hline Diabetes with complications & $32(14)$ & $171(8.1)$ & $203(8.6)$ & $0.002 *$ \\
\hline Diabetes without complications & $33(14.5)$ & $109(5.1)$ & $142(6)$ & $<0.001^{*}$ \\
\hline Asthma & $12(5.3)$ & $107(5)$ & $119(5.1)$ & 0.884 \\
\hline Chronic neurological disorder & $22(9.6)$ & $81(3.8)$ & $103(4.4)$ & $<0.001^{*}$ \\
\hline Hyperlipidemia & $4(1.8)$ & $92(4.3)$ & $96(4.1)$ & 0.061 \\
\hline Chronic kidney disease & $17(7.5)$ & $63(3)$ & $80(3.4)$ & $<0.001^{*}$ \\
\hline Chronic pulmonary disease & $11(4.8)$ & $57(42.7)$ & $68(2.9)$ & 0.067 \\
\hline Chronic hematologic disease & $9(3.9)$ & $40(1.9)$ & $49(2.1)$ & $0.038^{*}$ \\
\hline Malignant neoplasm & $11(4.8)$ & $38(1.8)$ & $49(2.1)$ & $0.002 *$ \\
\hline Hypothyroid & $6(2.6)$ & $43(2)$ & $49(2.1)$ & 0.543 \\
\hline Rheumatologic disorder & $5(2.2)$ & $27(1.3)$ & $32(1.4)$ & 0.254 \\
\hline Moderate or severe liver disease & $9(3.9)$ & $23(1.1)$ & $32(1.4)$ & $<0.001^{*}$ \\
\hline Fauvism & $6(2.6)$ & $15(0.7)$ & $21(0.9)$ & $0.0031^{*}$ \\
\hline Obesity & $2(0.9)$ & $5(0.2)$ & $7(0.3)$ & 0.091 \\
\hline Hyperthyroid & $1(0.4)$ & $4(0.2)$ & $5(0.2)$ & 0.436 \\
\hline HIV/AIDS & $2(0.9)$ & $4(0.2)$ & $6(0.3)$ & 0.05 \\
\hline Dementia & $3(1.3)$ & $4(0.2)$ & $7(0.3)$ & $0.003^{*}$ \\
\hline Mild liver disease & - & $4(0.2)$ & $4(0.2)$ & 0.665 \\
\hline Malnutrition & - & - & - & - \\
\hline Smoking, Yes & $17(7.5)$ & $111(5.2)$ & $128(5.4)$ & 0.159 \\
\hline Opium, Yes & $10(4.4)$ & $48(2.3)$ & $58(2.5)$ & $0.049 *$ \\
\hline Hookah, Yes & $4(1.8)$ & $33(1.6)$ & $37(1.6)$ & 0.818 \\
\hline Alcohol, Yes & $1(0.4)$ & $7(0.3)$ & $8(0.3)$ & 0.788 \\
\hline Methadone, Yes & $4(1.8)$ & $16(0.8)$ & $20(0.9)$ & 0.118 \\
\hline Other relevant risk factors & $45(19.7)$ & $193(9.1)$ & $238(10.1)$ & $<0.001^{*}$ \\
\hline \multicolumn{5}{|l|}{ Sign and symptoms } \\
\hline Cough & $88(38.6)$ & $1255(59.1)$ & $1343(57.1)$ & $0.001^{*}$ \\
\hline With sputum & $10(4.4)$ & $194(9.1)$ & $204(8.7)$ & $0.015 *$ \\
\hline Bloody sputum & $1(0.4)$ & $20(0.9)$ & $21(0.9)$ & 0.443 \\
\hline Shortness of breath & $138(60.5)$ & $1086(51.2)$ & $1224(52.1)$ & $0.007^{*}$ \\
\hline Fever & $82(36)$ & $978(46.1)$ & $1060(45.1)$ & $0.004^{*}$ \\
\hline Fatigue & $61(26.8)$ & $364(17.1)$ & $425(18.1)$ & $<0.0001 *$ \\
\hline Muscle aches & $35(15.4)$ & $382(18)$ & $417(17.7)$ & 0.321 \\
\hline Chills & $19(8.3)$ & $246(11.6)$ & $265(11.3)$ & 0.14 \\
\hline Vomiting & $18(7.9)$ & $204(9.6)$ & $222(9.4)$ & 0.4 \\
\hline Headache & $9(3.9)$ & $193(9.1)$ & $202(8.6)$ & $0.008 *$ \\
\hline Chest pain & $10(4.4)$ & $140(6.6)$ & $150(6.4)$ & 0.195 \\
\hline Diarrhea & $11(4.8)$ & $122(5.7)$ & $133(5.7)$ & 0.567 \\
\hline Sore throat & $9(3.9)$ & $117(5.5)$ & $126(5.4)$ & 0.319 \\
\hline Joint pain & $3(1.3)$ & $73(3.4)$ & $76(3.2)$ & 0.085 \\
\hline Lost sense (smell or taste) & $1(0.4)$ & $88(4.1)$ & $89(3.8)$ & $0.005^{*}$ \\
\hline Abdominal pain & $9(3.9)$ & $61(2.9)$ & $70(3)$ & 0.365 \\
\hline Vertigo & $1(0.4)$ & $60(2.8)$ & $61(2.6)$ & $0.031^{*}$ \\
\hline
\end{tabular}


Table 2. Continues.

\begin{tabular}{|c|c|c|c|c|}
\hline \multirow{2}{*}{ Variables } & $\begin{array}{c}\text { ICU Patients } \\
(\mathrm{n}=\mathbf{2 2 8})\end{array}$ & $\begin{array}{c}\text { Non-ICU Patients } \\
(n=2123)\end{array}$ & $\begin{array}{c}\text { Total Patients } \\
(\mathrm{n}=2351)\end{array}$ & \multirow{2}{*}{$P$ Value } \\
\hline & No. $(\%)$ & No. $(\%)$ & No. $(\%)$ & \\
\hline Runny nose & $3(1.3)$ & $38(1.8)$ & $41(1.7)$ & 0.603 \\
\hline Sweating & $4(1.8)$ & $22(1)$ & $26(1.1)$ & 0.324 \\
\hline Seizures & $8(3.5)$ & $5(0.2)$ & $13(0.6)$ & $0.001 *$ \\
\hline Altered consciousness & $2(0.9)$ & $8(0.4)$ & $10(0.4)$ & 0.270 \\
\hline Wheezing & - & $4(0.2)$ & $4(0.2)$ & 0.665 \\
\hline Conjunctivitis & - & $4(0.2)$ & $4(0.2)$ & 0.665 \\
\hline Palpitation & - & $5(0.2)$ & $5(0.2)$ & 0.6 \\
\hline Bleeding & - & $4(0.2)$ & $4(0.2)$ & 0.665 \\
\hline Lymphadenopathy & - & $2(0.1)$ & $2(0.1)$ & 0.815 \\
\hline Skin rash & - & $1(0)$ & $1(0)$ & 0.903 \\
\hline Skin ulcers & - & $1(0)$ & $1(0)$ & 0.903 \\
\hline Ear pain & - & $1(0)$ & - & .903 \\
\hline Lower chest wall in-drawing & - & - & - & - \\
\hline
\end{tabular}

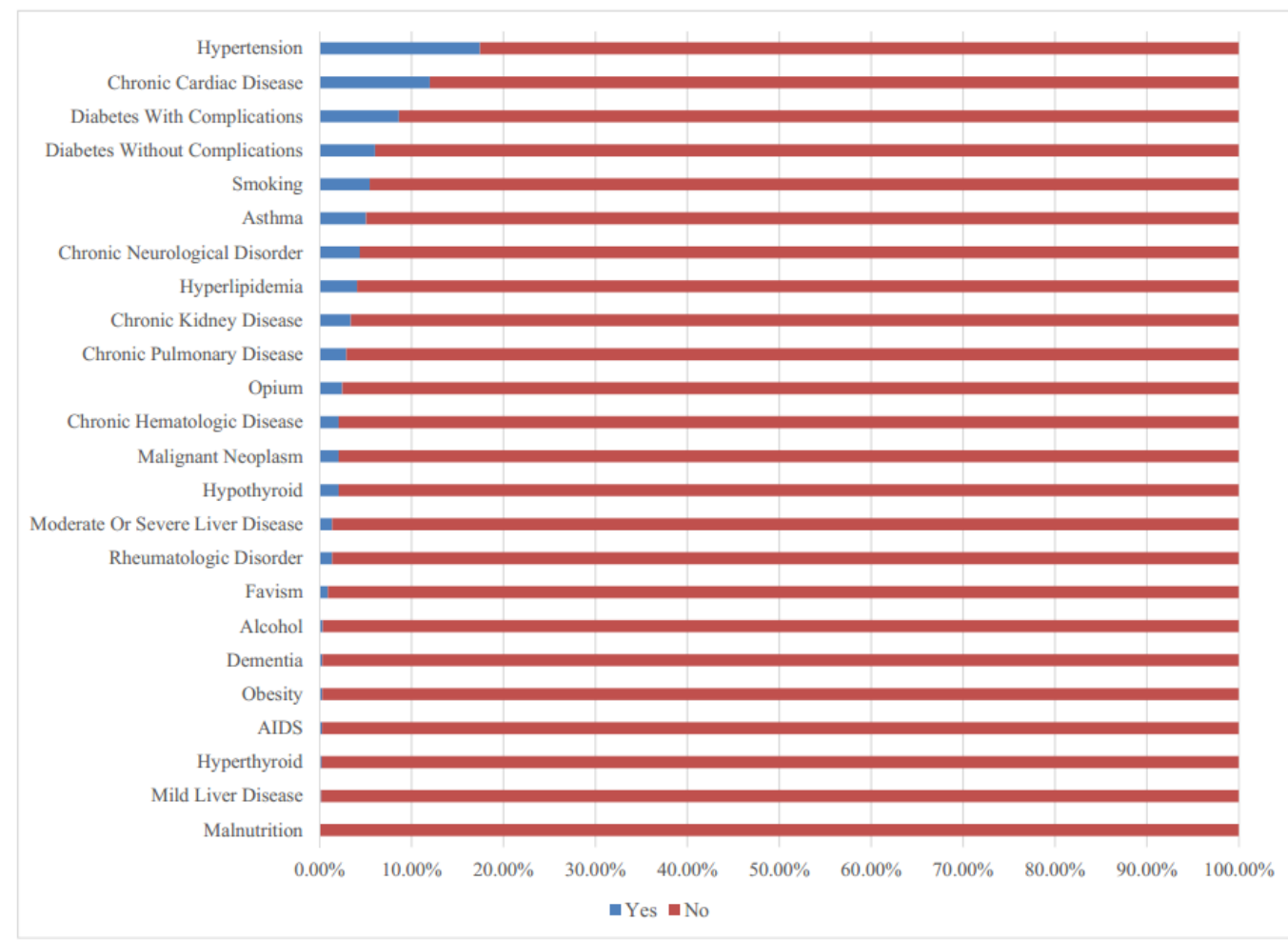

Figure 2. Distribution of Comorbidities among Patients.

patients. These procedures were respectively provided for $40.8 \%$ and $54.8 \%$ of ICU patients $(P<0.05)$. The duration of ventilation was 4.33 days on the average (1.79 days for non-ICU, and 5 days for ICU patients). The most common medications were antibiotics (80.1\%), and antiviral agents $(82.5 \%)$. The most frequently prescribed antiviral agents were hydroxychloroquine (63\%), lopinavir/ritonavir (24\%), and oseltamivir (18.2\%). Corticosteroids were used for $58.8 \%$ of ICU patients, but $19.4 \%$ of non-ICU patients
$(P<0.0001)$ (Table 4$)$.

\section{Outcomes}

Totally, $87.5 \%$ of the patients were discharged from the hospitals in good conditions and $12.5 \%$ passed away. Comparing ICU and non-ICU patients in terms of outcome showed that $35.5 \%$ of ICU patients were discharged while $64.5 \%$ of them passed away, both of which were significant compared to non-ICU patients $(P<0.0001)$. Non-ICU 
Table 3. Vital Signs and Laboratory Results on Admission of Hospitalized Patients Infected With COVID-19

\begin{tabular}{|c|c|c|c|c|c|}
\hline & & $\begin{array}{l}\text { ICU Patients } \\
(n=228)\end{array}$ & $\begin{array}{c}\text { Non-ICU Patients } \\
(n=2123)\end{array}$ & $\begin{array}{c}\text { Total Patients } \\
(n=2351)\end{array}$ & $P$ Value \\
\hline \multirow{2}{*}{ Temperature } & Mean \pm SD & $37.21 \pm 0.86$ & $37.27 \pm 0.78$ & $37.27 \pm 0.79$ & 0.118 \\
\hline & Median & 37.0 & 37.0 & 37.0 & 0.102 \\
\hline \multirow{2}{*}{ HR } & Mean \pm SD & $89.67 \pm 24.28$ & $83.98 \pm 16.46$ & $84.53 \pm 17.44$ & $0.008^{*}$ \\
\hline & Median & 84.5 & 82.0 & 82.0 & 0.119 \\
\hline \multirow{2}{*}{ RR } & Mean \pm SD & $23.44 \pm 11.96$ & $19.88 \pm 5.45$ & $20.24 \pm 6.50$ & $<0.0001^{*}$ \\
\hline & Median & 20.0 & 19.0 & 19.0 & $<0.0001^{*}$ \\
\hline \multirow{2}{*}{ Systolic BP } & Mean \pm SD & $121.7 \pm 24.43$ & $118.89 \pm 18.87$ & $119.18 \pm 19.52$ & 0.221 \\
\hline & Median & 120.0 & 120.0 & 120.0 & $0.001^{*}$ \\
\hline \multirow{2}{*}{ Diastolic BP } & Mean \pm SD & $74.39 \pm 13.77$ & $74.56 \pm 11.49$ & $74.55 \pm 11.69$ & 0.859 \\
\hline & Median & 71.0 & 75.0 & 75.0 & 0.811 \\
\hline \multirow{2}{*}{ Oxygen Saturation } & Mean \pm SD & $88.18 \pm 11.96$ & $94.85 \pm 4.91$ & $94.17 \pm 6.36$ & $<0.0001^{*}$ \\
\hline & Median & 91.0 & 96.00 & 96.0 & $<0.0001^{*}$ \\
\hline \multicolumn{6}{|l|}{ Lab tests } \\
\hline \multirow{2}{*}{ Hemoglobin } & Mean \pm SD & $11.28 \pm 2.32$ & $12.25 \pm 2.16$ & $12.15 \pm 2.198$ & $<0.0001^{*}$ \\
\hline & Median & 11.1 & 12.3 & 12.2 & $<0.0001^{*}$ \\
\hline \multirow{2}{*}{ WBC } & Mean \pm SD & $10.96 \pm 9.01$ & $7.48 \pm 4.92$ & $7.86 \pm 5.61$ & $<0.0001^{*}$ \\
\hline & Median & 9.25 & 6.5 & 6.7 & $<0.0001^{*}$ \\
\hline \multirow{2}{*}{ Lymphocyte } & Mean \pm SD & $16.22 \pm 12.95$ & $24.87 \pm 13.04$ & $23.97 \pm 13.29$ & $<0.0001^{*}$ \\
\hline & Median & 12.3 & 23.3 & 22 & $<0.0001^{*}$ \\
\hline \multirow{2}{*}{ Neutrophil } & Mean \pm SD & $77.10 \pm 15.79$ & $66.9 \pm 15.39$ & $68 \pm 15.75$ & $<0.0001^{*}$ \\
\hline & Median & 82.0 & 68.2 & 69.8 & $<0.0001^{*}$ \\
\hline \multirow{2}{*}{ Hematocrit } & Mean \pm SD & $35.21 \pm 6.86$ & $37.52 \pm 17.11$ & $37.28 \pm 16.34$ & $<0.0001^{*}$ \\
\hline & Median & 35.2 & 37.2 & 37 & $0.003^{*}$ \\
\hline \multirow{2}{*}{ Platelets } & Mean \pm SD & $223.21 \pm 119.94$ & $230.20 \pm 99.89$ & $229.46 \pm 102.19$ & 0.10 \\
\hline & Median & 200.5 & 211.0 & 210 & 0.101 \\
\hline \multirow{2}{*}{ APTT } & Mean \pm SD & $36.46 \pm 14.66$ & $34.10 \pm 10.0$ & $34.37 \pm 10.67$ & 0.151 \\
\hline & Median & 34.0 & 33.0 & 33 & 0.277 \\
\hline \multirow{2}{*}{ PT } & Mean \pm SD & $15.15 \pm 5.89$ & $13.59 \pm 2.73$ & $13.81 \pm 3.42$ & $<0.0001^{*}$ \\
\hline & Median & 13.20 & 13.0 & 13.0 & $0.003 *$ \\
\hline \multirow{2}{*}{ INR } & Mean \pm SD & $1.29 \pm 0.7$ & $1.11 \pm 0.41$ & $1.14 \pm 0.47$ & $0.001 *$ \\
\hline & Median & 1.05 & 1.0 & 1.0 & 0.205 \\
\hline \multirow{2}{*}{ ALT } & Mean \pm SD & $64.80 \pm 102.04$ & $45.08 \pm 99.12$ & $47.36 \pm 99.62$ & $0.015^{*}$ \\
\hline & Median & 34.0 & 31.0 & 32.0 & 0.065 \\
\hline \multirow{2}{*}{ Total Bilirubin } & Mean \pm SD & $2.39 \pm 4.19$ & $1.37 \pm 2.74$ & $1.51 \pm 2.99$ & $<0.0001^{*}$ \\
\hline & Median & 1.1 & 0.7 & 0.8 & $<0.0001^{*}$ \\
\hline \multirow{2}{*}{ AST } & Mean \pm SD & $94.41 \pm 179.96$ & $57.73 \pm 316.8$ & $61.95 \pm 304.39$ & $<0.001 *$ \\
\hline & Median & 75.0 & 33.0 & 34 & $<0.0001^{*}$ \\
\hline \multirow{2}{*}{ Glucose } & Mean \pm SD & $170.47 \pm 115.28$ & $124.13 \pm 72.02$ & $130.75 \pm 81.23$ & $<0.001 *$ \\
\hline & Median & 138.0 & 103.0 & 105 & $<0.0001^{*}$ \\
\hline \multirow{2}{*}{ Blood Urea Nitrogen } & Mean \pm SD & $45.25 \pm 40.58$ & $27.01 \pm 25.68$ & $29.08 \pm 28.38$ & $<0.0001^{*}$ \\
\hline & Median & 30.0 & 21.0 & 22 & $<0.0001^{*}$ \\
\hline \multirow{2}{*}{ Creatinine } & Mean \pm SD & $1.49 \pm 1.70$ & 1.071 .04 & $1.12 \pm 1.15$ & $<0.0001^{*}$ \\
\hline & Median & 1.0 & 0.9 & 0.9 & $<0.0001^{*}$ \\
\hline म & Mean \pm SD & $793.15 \pm 854.57$ & $459.71 \pm 599.43$ & $492.75 \pm 636.76$ & $<0.001 *$ \\
\hline LDா & Median & 529.0 & 373.0 & 385.5 & $<0.0001^{*}$ \\
\hline Codium & Mean \pm SD & $138.89 \pm 7.72$ & $138.15 \pm 5.75$ & $138.23 \pm 6.01$ & 0.628 \\
\hline sodium & Median & 138.0 & 138.0 & 138.0 & 0.656 \\
\hline
\end{tabular}


Table 3. Continues.

\begin{tabular}{lccccc}
\hline & & $\begin{array}{c}\text { ICU Patients } \\
(\boldsymbol{n}=\mathbf{2 2 8})\end{array}$ & $\begin{array}{c}\text { Non-ICU Patients } \\
(\boldsymbol{n}=\mathbf{2 1 2 3})\end{array}$ & $\begin{array}{c}\text { Total Patients } \\
(\boldsymbol{n}=\mathbf{2 3 5 1})\end{array}$ & $\boldsymbol{P}$ Value \\
\hline Potassium & Mean \pm SD & $4.27 \pm 0.71$ & $4.23 \pm 1.68$ & $4.24 \pm 1.59$ & 0.227 \\
& Median & 4.10 & 4.10 & 4.10 & 0.517 \\
Troponin & Mean \pm SD & $250.12 \pm 724.17$ & $40.21 \pm 320.103$ & $70.31 \pm 409.64$ & $<0.0001^{*}$ \\
& Median & 14.0 & 2.0 & 3.0 & $<0.0001^{*}$ \\
Mg & Mean \pm SD & $2.30 \pm 0.72$ & $1.94 \pm 0.25$ & $2.03 \pm 0.44$ & $<0.0001^{*}$ \\
& Median & 2.1 & 2.0 & 2.0 & $0.001^{*}$ \\
ESR & Mean \pm SD & $41.27 \pm 30.55$ & $35.02 \pm 27.04$ & $35.61 \pm 27.44$ & $0.043^{*}$ \\
& Median & 37.0 & 29.0 & 30.0 & $<0.0001^{*}$ \\
\hline
\end{tabular}

*Significant difference

Table 4. Treatments and Medications for Hospitalized Patients Infected with COVID-19

\begin{tabular}{|c|c|c|c|c|}
\hline \multirow[t]{2}{*}{ Variables } & $\begin{array}{c}\text { ICU Patients } \\
(\mathrm{n}=\mathbf{2 2 8})\end{array}$ & $\begin{array}{c}\text { Non-ICU Patients } \\
(n=2123)\end{array}$ & $\begin{array}{c}\text { Total Patients } \\
(\mathrm{n}=2351)\end{array}$ & \multirow[t]{2}{*}{$P$ Value } \\
\hline & No. $(\%)$ & No. $(\%)$ & No. $(\%)$ & \\
\hline Oxygen therapy & $218(95.6)$ & $890(41.9)$ & $1108(47.1)$ & $<0.001^{*}$ \\
\hline Noninvasive ventilation & $93(40.8)$ & $12(0.6)$ & $105(4.5)$ & $0.001 *$ \\
\hline Invasive ventilation & $125(54.8)$ & $34(1.6)$ & $159(6.8)$ & $<0.001^{*}$ \\
\hline \multicolumn{5}{|c|}{ Invasive ventilation duration } \\
\hline Mean $( \pm S D)$, Day & $5.01 \pm 5.6$ & $1.79 \pm 1.75$ & $4.33 \pm 5.23$ & $<0.001^{*}$ \\
\hline Median (Q1, Q3) & $3(1.25,7)$ & $1(1,1.5)$ & $2(1,5)$ & - \\
\hline Min, Max & 1,40 & 1,8 & 1,40 & - \\
\hline \multicolumn{5}{|l|}{ Medications } \\
\hline Any antibiotics & $213(93.4)$ & 1669 (78.6) & $1882(80.1)$ & $0.007^{*}$ \\
\hline Any antiviral agent & $205(89.9)$ & $1734(81.7)$ & 1939 (82.5) & $0.001^{*}$ \\
\hline Hydroxychloroquine & $129(56.6)$ & $1352(63.7)$ & $1481(63)$ & $<0.001^{*}$ \\
\hline Lopinavir/Ritonavir & $105(46.1)$ & $460(21.7)$ & $565(24.0)$ & $<0.001^{*}$ \\
\hline Oseltamivir & $14(14)$ & 395 (18.6) & $427(18.2)$ & $0.003 *$ \\
\hline Interferon Beta & $78(34.2)$ & $303(14.3)$ & $381(16.2)$ & $<0.001^{*}$ \\
\hline Atazanavir & $49(21.5)$ & $192(9)$ & $241(10.3)$ & $<0.001 *$ \\
\hline Ribavirin & $11(4.8)$ & $21(1)$ & $32(1.4)$ & $<0.001^{*}$ \\
\hline Remdesivir & $9(3.9)$ & $3(0.1)$ & $12(0.5)$ & $<0.001^{*}$ \\
\hline Albumin & $15(6.6)$ & $1(0)$ & $16(0.7)$ & $<0.001^{*}$ \\
\hline Corticosteroid & $134(58.8)$ & 411 (19.4) & $545(23.2)$ & $<0.001^{*}$ \\
\hline Plasma exchange & $22(9.6)$ & $6(0.3)$ & $28(1.2)$ & $<0.001^{*}$ \\
\hline IVIg & $33(14.5)$ & $22(1)$ & $55(2.3)$ & $<0.001^{*}$ \\
\hline
\end{tabular}

*Significant difference

Table 5. Clinical Outcomes of Hospitalized Patients Infected with COVID-19

\begin{tabular}{lcccc}
\hline \multirow{2}{*}{ Variables } & ICU patients $(\mathbf{n}=\mathbf{2 2 8})$ & Non-ICU Patients $(\mathbf{n = 2 1 2 3})$ & Total Patients $(\mathbf{n}=\mathbf{2 3 5 1})$ & \multirow{P}{*}{$\boldsymbol{P}$ Value } \\
\cline { 2 - 4 } & No. $(\mathbf{\%})$ & No. $(\%)$ & No. $(\%)$ & \\
\hline Discharged alive & $81(35.5)$ & $1975(93)$ & $2056(87.5)$ & $<0.001^{*}$ \\
Death & $147(64.5)$ & $148(7)$ & $295(12.5)$ & \\
LOS, day & & & & $<0.001^{*}$ \\
Mean $( \pm$ SD) & $8.51 \pm 6.97$ & $4.03 \pm 3.46$ & $4.47 \pm 4.17$ & $<0.001^{*}$ \\
Median (Q1, Q3) & $7(3.25,11.75)$ & $3(2,5)$ & $3(2,6)$ & - \\
Min, Max & 1,43 & 1,39 & 1,43 & - \\
\hline
\end{tabular}

*Significant difference 


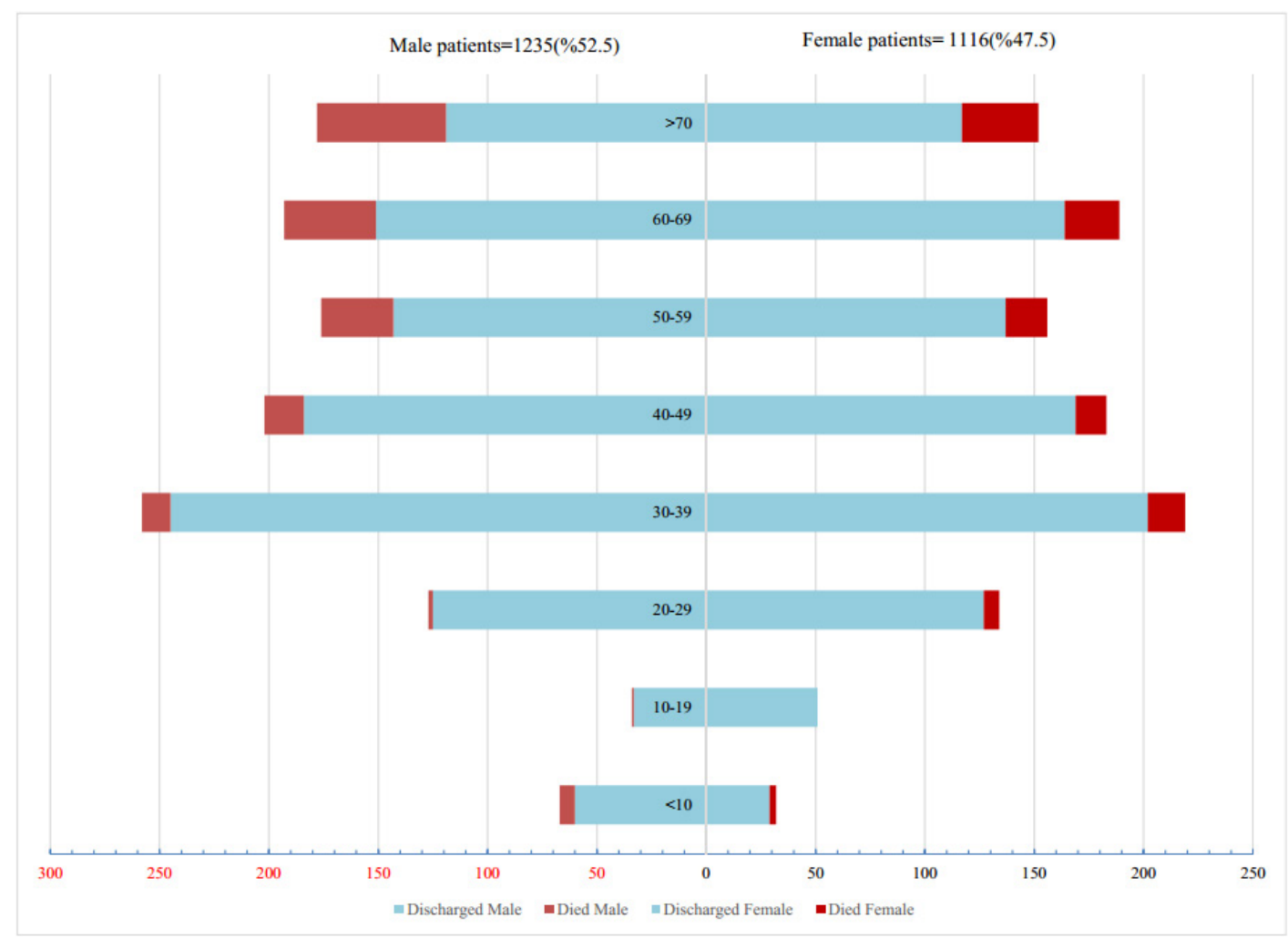

Figure 3. Distribution of Outcomes between Men and Women Classified by Age Groups.

patients were hospitalized for 4 days on the average while it was 8.5 days for ICU patients. The average ICU length of stay was 6.35 ( \pm 6.2 ) days $(\operatorname{Min}=1, \operatorname{Max}=43$, Median $=4$, $\mathrm{Q} 1=2, \mathrm{Q} 3=8$ ) (Table 5). Figure 3 shows the distribution of outcomes (discharge and death) between male and female patients classified by age groups. As is shown, in both sexes, more deaths occurred among patients in the age groups of more than $70,60-69$, and 50-59 years, in decreasing order of frequency.

\section{Discussion}

The present study is the first descriptive study addressing clinical and epidemiological characteristics of COVID-19 in southern Iran. The mean and median age for our patients was 47 and 46 years, respectively. In a study from China, the mean age and the most frequent age groups were 55.5, and 50-59 years (30\%), respectively. ${ }^{10}$ Studies from the UK, Germany and Italy reported median age values of 73, 73 and 71 years, respectively. ${ }^{7-8,15} \mathrm{~A}$ study in Tehran, Iran, showed that the mean age was 55.5, and the majority of patients were in the age group of 50-70 years. ${ }^{21}$ This indicates that our patients are younger. Our ICU patients were significantly older than non-ICU patients (53 vs. 46 years). Another study showed that there was no difference between ICU and non-ICU patients in terms of patients' age ${ }^{12}$ and another reported that ICU patients were older. ${ }^{22}$ We found that this disease infected men more than women. Furthermore, male patients were hospitalized in the ICU more than women. Other studies have reported similar results..$^{7-8,10,21}$ Some studies have suggested that this might be related to the $\mathrm{X}$ chromosome and sex hormones as factors for stimulating adaptive immunity. ${ }^{10}$

Most of our cases suffered from comorbidities including hypertension, chronic cardiac diseases, diabetes, and asthma. Our findings confirm earlier studies. ${ }^{8,21,23-24}$ A study from China reported cardiovascular and cerebrovascular diseases (40\%), endocrine system diseases such as diabetes (13\%), and digestive system disease (11\%) as common comorbidities..$^{10}$ A UK study reported that the most common comorbidities were chronic cardiac disease $(30.9 \%)$, diabetes without complications (20.7\%), chronic pulmonary disease excluding asthma (17.7\%), chronic kidney disease (16.2\%), and asthma (14.5\%), which was almost similar to our study in terms of common comorbidities. However, our patients suffered from fewer comorbidities than the UK report. ${ }^{7}$ A systematic review on 10 articles and 76,993 patients indicated that hypertension (16.37\%), cardiovascular disease $(12.11 \%)$, smoking history $(7.63 \%)$, and diabetes $(7.87 \%)$ were the most common comorbidities in people infected with COVID-19. Chronic obstructive pulmonary disease (COPD), malignancy, and chronic kidney disease were other common comorbidities. ${ }^{25}$ Another systematic review shows that hypertension (15.6\%), diabetes (7.7\%), cardiovascular disease $(4.7 \%)$, malignancy $(1.2 \%)$ were the most prevalent comorbidities. ${ }^{26}$ These studies confirm our findings.

We found that hypertension, chronic cardiac disease, diabetes, chronic neurological disorders, chronic kidney disease, chronic hematologic disease, malignant neoplasm, 
moderate or severe liver disease, dementia and fauvism were more common in ICU patients than non-ICU patients. Another study showed that diabetes, hypertension and cardiovascular disease were the most common comorbidities and there were no differences between ICU and non-ICU patients in this respect ${ }^{12}$; however, a study reported that hypertension, cardiovascular disease, diabetes, and cerebrovascular disease were significantly more common in ICU patients. ${ }^{22}$

In our patients, the most common reported symptoms were cough, shortness of breath, fever, fatigue, and muscle aches. Chen reported fever (83\%), cough (82\%), shortness of breath (31\%), and muscle ache (11\%) as common symptoms of patients in China. ${ }^{10} \mathrm{~A}$ recent UK study confirms our findings and presents cough (68.9\%), fever (71.6), and shortness of breath (71.2\%) as the most common symptoms. ${ }^{7}$ A systematic review analyzed 43 studies involving 3600 patients found that fever (83.3\%), cough $(60.3 \%)$, and fatigue (38.0\%) were the most common clinical symptoms. ${ }^{27}$ Another systematic review indicated that fever $(85.6 \%)$, cough $(65.7 \%)$, fatigue $(42.4 \%)$, and dyspnea (21.4\%) were the most common symptoms. ${ }^{26} \mathrm{~A}$ systematic review also showed that fever (88.7\%), cough (57.6\%), and dyspnea (45.6\%) were the most common symptoms. ${ }^{28}$ These studies are almost similar to our study.

We also found that only cough, seizure, shortness of breath, fever, fatigue, headache, lost sense (of smell or taste), and vertigo were significantly different between ICU and non-ICU patients. According to a study, only dyspnea was significantly more common in ICU patients ${ }^{12}$; however, another study reported significantly higher anorexia, dyspnea, pharyngalgia, dizziness, and abdominal pain among ICU patients. ${ }^{22}$

In our study, $47.1 \%$ of the patients $(95.6 \%$ of ICU patients) received oxygen therapy. Only $4.5 \%$ and $6.8 \%$ of patients received non-invasive and invasive ventilation, respectively. Antibiotics (80.1\%) and antiviral agents $(82.5 \%)$ were common medications. In the UK, 55\% received high flow oxygen, while $16 \%$, and $10 \%$ received non-invasive and invasive ventilation, respectively. ${ }^{7}$ Similarly, $76 \%$ of patients reported in a Chinese study received oxygen therapy. Non-invasive and invasive ventilation were used for $13 \%$ and $4 \%$, respectively. Also, $71 \%$ and $76 \%$ of patients received antibiotics and antiviral treatment, respectively. ${ }^{10}$ The use of antibiotic therapy (100\%) and antiviral therapy (93\%) reported in another study is similar to our findings. The authors also reported the use of non-invasive ventilation $(24 \%)$ or invasive mechanical ventilation (5\%) among Chinese patients. ${ }^{12}$ Prescribing antibiotics for our patients was similar to an Italian study. $^{8}$

In our study, $9.7 \%$ of patients were hospitalized in ICU. It was lower than New York (14.2\%), ${ }^{13}$ the UK $(17 \%),{ }^{7}$ France $(31 \%),{ }^{14}$ Italy $(20.5 \%)^{8}$ and Germany $(21 \%) .{ }^{15}$ According to a systematic review, $20.3 \%$ of patients required ICU services. ${ }^{28}$ This study may indicate that our patients were infected with a less severe disease. Additionally, the mortality rate was $12.5 \%$ among all patients and $64.5 \%$ among ICU patients. Mortality was higher among older male patients. The mortality was reported at $11 \%$ and $15 \%$ in Chinese studies, ${ }^{10,12} 20 \%$ in France, ${ }^{14} 26 \%$ in the $\mathrm{UK}^{7}, 43.6 \%$ in Italy ${ }^{8}$ and $16.6 \%$ in Germany. ${ }^{15}$ A systematic review indicates that $13.9 \%$ of hospitalized patients have fatal outcomes and the case fatality rate is $>13 \%{ }^{28}$ In a study from Tehran, the case fatality rate was $8.06 \%$ among hospitalized patients and most deceased patients were $\geq 60$ years of age (mean $=65.38$ ). Additionally, the fatality rate was $8.54 \%$ and $7.13 \%$ for men and women, respectively. ${ }^{21}$ Although the age of patients who died was almost similar in our and the $\mathrm{UK}^{7}$ and Tehran ${ }^{21}$ studies, mortality was lower in our population. Conversely, in the UK, $32 \%$ of patients who received a high level of care such as ICU services died, while this rate was $64.5 \%$ in our population, which was higher than the $\mathrm{UK}^{7}$, Italy $(26 \%),{ }^{29}$ China (38\%) $)^{12}$ and Germany (29\%). ${ }^{15}$

This study had some strengths. The data was extracted from a multi-center registry with considerable quality control. This registry is currently enrolling patients and enables us to analyze more patients in the future and compare the trends. Furthermore, this registry applied ISARIC case report form that improved the comparability of findings. Despite these strengths, some limitations should be considered. Our analysis did not include homequarantined cases and outpatients. Although we collected data regarding outpatients, these data were not complete and were under the quality improvement process. Therefore, we excluded these patients from the study.

In conclusion, this study is the first epidemiologic investigation with quite a high sample size in southern Iran. It shows that hypertension, chronic cardiac disease, diabetes, and asthma are the most prevalent comorbidities, and cough, shortness of breath, fever, fatigue, and muscle aches are the most common signs and symptoms. ICU and non-ICU patients are different in terms of some comorbidities and symptoms. The mortality rate is $12.5 \%$ in general and $64.5 \%$ in ICU patients and is higher in older men. These findings may play a major role in healthcare policy-making for this disease.

\section{Authors' Contributions}

$\mathrm{MH}$ : Conceptualization, data curation, methodology, writingthe original draft, validation, writing - review and editing, project administration, resources, supervision. FK: Conceptualization, data curation, methodology, software, visualization, writingthe original draft, validation, writing - review and editing, project administration, resources, supervision. MK, GM and NA: Data curation; Methodology; Writing - review and editing. MS and ND: Methodology; Writing- the original draft; Writing - review and editing. AS: Conceptualization, formal analysis, methodology, software, writing- the original draft, validation, writing - review and editing, supervision.

\section{Conflict of Interest Disclosures}

There are no conflicts of interest. 


\section{Ethical Statement}

This study received ethical approval from the Ethics Committee of Hormozgan University of Medical Sciences (HUMS. REC.1398.482).

\section{Funding}

This study was financially supported by a grant provided by Hormozgan University of Medical Sciences (Main project number at HUMS-Vice Chancellor for Research and Technology: 980464). The funder had no role in the design and conduct of the study; collection, management, analysis, and interpretation of the data; preparation, review, or approval of the manuscript; and decision to submit the manuscript for publication.

\section{Acknowledgements}

We would like to express our gratitude to Dr. Teamur Aghamolaei (the HUMS Vice-chancellor for Research and Technology) and all the staff of Health Information Management Departments at healthcare facilities affiliated to HUMS who supported us to collect data.

\section{References}

1. Wang LS, Wang YR, Ye DW, Liu QQ. A review of the 2019 Novel Coronavirus (COVID-19) based on current evidence. Int J Antimicrob Agents. 2020;55(6):105948. doi:10.1016/j. ijantimicag.2020.105948

2. Petrosillo N, Viceconte G, Ergonul O, Ippolito G, Petersen E. COVID-19, SARS and MERS: are they closely related? Clin Microbiol Infect. 2020; 26(6):729-34. doi:10.1016/j. cmi.2020.03.026

3. Aggarwal S, Garcia-Telles N, Aggarwal G, Lavie C, Lippi G, Henry BM. Clinical features, laboratory characteristics, and outcomes of patients hospitalized with coronavirus disease 2019 (COVID-19): Early report from the United States. Diagnosis (Berl). 2020;7(2):91-6. doi:10.1515/dx2020-0046

4. Siordia JA, Jr. Epidemiology and clinical features of COVID-19: A review of current literature. J Clin Virol. 2020;127:104357. doi:10.1016/j.jcv.2020.104357

5. World Heahlth Organization.WHO Coronavirus (COVID-19) Dashboard: WHO; 2020. Available from: https://covid19.who.int/. Accessed March 4, 2020.

6. Khorrami F, Shahi M, DavariDolatabadi N, Alishan Karami $\mathrm{N}$, HasaniAzad M, Jafariyan F, et al. Implementation of regional COVID-19 registry in Hormozgan (RCovidRH), Iran: Rationale and study protocol. Med J Islam Repub Iran. 2020;34:96. doi: 10.34171/mjiri.34.96.

7. Docherty $A B$, Harrison EM, Green CA, Hardwick HE, Pius R, Norman L, et al. Features of 20133 UK patients in hospital with covid-19 using the ISARIC WHO clinical characterisation protocol: prospective observational cohort study. BMJ. 2020;369:m1985. doi:10.1136/bmj.m1985

8. Vena A, Giacobbe DR, Di Biagio A, Mikulska M, Taramasso L, De Maria A, et al. Clinical characteristics, management and in-hospital mortality of patients with coronavirus disease 2019 in Genoa, Italy. Clin Microbiol Infect. 2020;26(11):1537-44. doi:10.1016/j.cmi.2020.07.049

9. Guan WJ, Ni ZY, Hu Y, Liang WH, Ou CQ, He JX, et al. Clinical characteristics of Coronavirus Disease 2019 in China. N Engl J Med. 2020;382(18):1708-20. doi:10.1056/ NEJMoa2002032

10. Chen N, Zhou M, Dong X, Qu J, Gong F, Han Y, et al.
Epidemiological and clinical characteristics of 99 cases of 2019 novel coronavirus pneumonia in Wuhan, China: a descriptive study. Lancet. 2020;395(10223):507-13. doi:10.1016/s0140-6736(20)30211-7

11. Chen P, Zhang Y, Wen Y, Guo J, Jia J, Ma Y, et al. Epidemiological and clinical characteristics of 136 cases of COVID-19 in main district of Chongqing. J Formos Med Assoc. 2020;119(7):1180-4. doi:10.1016/j.jfma.2020.04.019

12. Huang C, Wang Y, Li X, Ren L, Zhao J, Hu Y, et al. Clinical features of patients infected with 2019 novel coronavirus in Wuhan, China. Lancet. 2020;395(10223):497-506. doi:10.1016/S0140-6736(20)30183-5

13. Richardson S, Hirsch JS, Narasimhan M, Crawford JM, McGinn T, Davidson KW, et al. Presenting characteristics, comorbidities, and outcomes among 5700 patients hospitalized with COVID-19 in the New York city area. JAMA. 2020;323(20):2052-9. doi:10.1001/jama.2020.6775

14. Faury H, Courboulès C, Payen M, Jary A, Hausfater P, Luyt C, et al. Medical features of COVID-19 and influenza infection: A comparative study in Paris, France. J Infect. 2021;82(2):e36-e9. doi:10.1016/j.jinf.2020.08.017

15. Nachtigall I, Lenga $P$, Jóźwiak K, Thürmann P, MeierHellmann A, Kuhlen R, et al. Clinical course and factors associated with outcomes among 1904 patients hospitalized with COVID-19 in Germany: an observational study. Clin Microbiol Infect. 2020;26(12):1663-9. doi:10.1016/j. cmi.2020.08.011

16. Maechler F, Gertler M, Hermes J, van Loon W, Schwab F, Piening B, et al. Epidemiological and clinical characteristics of SARS-CoV-2 infections at a testing site in Berlin, Germany, March and April 2020-a cross-sectional study. Clin Microbiol Infect. 2020;26(12):1685.e7-.e12. doi:10.1016/j.cmi.2020.08.017

17. International Severe Acute Respiratory and Emerging Infection Consortium. Novel coronavirus (ncov) acute respiratory infection clinical characterization data tool. 2020. Available from: https://isaric.tghn.org/covid-19clinical-research-resources. Accessed March 4, 2020.

18. World Health Organization. Interim case reporting form for 2019 Novel Coronavirus (2019-nCoV) of confirmed and probable cases: WHO Minimum Data Set Report Form: World Health Organization; 2020. Available from: https://www.who.int/docs/default-source/ coronaviruse/20200121-2019-ncov-reporting-form.pdf. Accessed March 4, 2020.

19. Centers for Disease Control and Prevention (CDC). Human Infection with 2019 Novel Coronavirus Person Under Investigation (PUI) and Case Report Form. 2020, USA: Center for Disease Prevention and Control; 2020. Available from: URL: https://www.cdc.gov/coronavirus/2019-ncov/ downloads/pui-form.pdf. Accessed March 4, 2020.

20. Zhu N, Zhang D, Wang W, Li X, Yang B, Song J, et al. A novel coronavirus from patients with pneumonia in China, 2019. N Engl J Med. 2020;382(8):727-33. doi:10.1056/ NEJMoa2001017

21. Nikpouraghdam M, Jalali Farahani A, Alishiri G, Heydari S, Ebrahimnia M, Samadinia H, et al. Epidemiological characteristics of coronavirus disease 2019 (COVID-19) patients in Iran: A single center study. J Clin Virol. 2020; 127:104378. doi:10.1016/j.jcv.2020.104378

22. Wang D, Hu B, Hu C, Zhu F, Liu X, Zhang J, et al. Clinical characteristics of 138 hospitalized patients with 2019 novel 
coronavirus-infected pneumonia in Wuhan, China. JAMA. 2020;323(11):1061-9. doi:10.1001/jama.2020.1585

23. Guan WJ, Liang WH, Zhao Y, Liang HR, Chen ZS, Li YM, et al. Comorbidity and its impact on 1590 patients with Covid-19 in China: A nationwide analysis. Eur Respir J. 2020;55(5):2000547. doi:10.1183/13993003.00547-2020

24. Li B, Yang J, Zhao F, Zhi L, Wang X, Liu L, et al. Prevalence and impact of cardiovascular metabolic diseases on COVID-19 in China. Clin Res Cardiol. 2020;109(5):531-8. doi:10.1007/s00392-020-01626-9

25. Emami A, Javanmardi F, Pirbonyeh N, Akbari A. Prevalence of underlying diseases in hospitalized patients with COVID-19: a systematic review and meta-analysis. Arch Acad Emerg Med. 2020;8(1):e35.

26. Hu Y, Sun J, Dai Z, Deng H, Li X, Huang Q, et al. Prevalence and severity of corona virus disease 2019 (COVID-19): A systematic review and meta-analysis. J Clin Virol.
2020;127:104371. doi:10.1016/j.jcv.2020.104371

27. Fu L, Wang B, Yuan T, Chen X, Ao Y, Fitzpatrick T, et al. Clinical characteristics of coronavirus disease 2019 (COVID-19) in China: A systematic review and metaanalysis. J Infect. 2020;80(6):656-65. doi:10.1016/j. jinf.2020.03.041

28. Rodriguez-Morales AJ, Cardona-Ospina JA, GutiérrezOcampo E, Villamizar-Peña R, Holguin-Rivera Y, EscaleraAntezana JP, et al. Clinical, laboratory and imaging features of COVID-19: A systematic review and meta-analysis. Travel Med Infect Dis. 2020;34:101623. doi:10.1016/j. tmaid.2020.101623

29. Grasselli G, Zangrillo A, Zanella A, Antonelli M, Cabrini L, Castelli A, et al. Baseline characteristics and outcomes of 1591 patients infected with SARS-CoV-2 admitted to ICUs of the Lombardy Region, Italy. JAMA. 2020;323(16):157481. doi:10.1001/jama.2020.5394 